\title{
A Correlation-Based Distance Function for Nearest Neighbor Classification
}

\author{
Yanet Rodriguez ${ }^{1}$, Bernard De Baets ${ }^{2}$, Maria M. Garcia ${ }^{1}$, \\ Carlos Morell ${ }^{1}$, and Ricardo Grau ${ }^{1}$ \\ ${ }^{1}$ Universidad Central de Las Villas, C. Camajuani km $5 \frac{1}{2}$, Santa Clara, Cuba \\ ${ }^{2}$ Ghent University, Coupure links 653, B-9000 Gent, Belgium ${ }^{\star}$
}

\begin{abstract}
The Nearest Neighbor rule is a well-known classification method largely studied in the pattern recognition community, both for its simplicity and its performance. The definition of the distance function is central for obtaining a good accuracy on a given data set and different distance functions have been proposed to increase the performance. This paper proposes a new distance function based on the correlation of fuzzy sets, called Fuzzy Correlation-based Difference Metric. The proposed distance function is a generalization of the Value Difference Metric and applies to both nominal and continuous attributes in a uniform way. Fuzzy sets are used to represent numeric attributes. A uninorm operator is used to aggregate local differences. Experimental results using an standard $k$-NN algorithm show a significant improvement in comparison to other distance functions proposed before.
\end{abstract}

Keywords: Nearest Neighbour Classification, Distance functions, Value Difference Metric, Fuzzy Sets Theory.

\section{Introduction}

The concept of similarity (or the dual concept of distance) plays a central role in some pattern recognition methods such as $k$-NN ( $k$-Nearest Neighbor). Distance functions can be categorized into those that can handle ordinal (continuous or discrete), nominal (or symbolic), and heterogeneous input data consisting of both ordinal and nominal data. Because the notion of distance is intrinsically numerical, most available distance are defined for data with ordinal attributes.

Two of the most common distance functions are the Euclidean distance and the Hamming distance. The Euclidean distance is restricted to ordinal and the latter one is usually used for nominal attributes. Some researchers have used the overlap metric for nominal attributes. The Value Difference Metric (VDM), which was introduced by Stanfill and Waltz in 1986 [1], is another appropriated distance function for nominal attributes. None of them are able to deal with heterogeneous data. Existing systems using the VDM either rely on discretization methods to convert continuous attributes into discrete ones (DVDM) or

\footnotetext{
* This work was supported in part by VLIR (Vlaamse InterUniversitaire Raad, Flemish Interuniversity Council, Belgium) under the IUC Program VLIR-UCLV.
} 
combine original continuous values by using interpolation (IVDM) [2]. There are other distance functions to deal with heterogeneous data, usually by combining the previous ones. For example the Heterogeneous Euclidean-Overlap Metric (HEOM) and the Heterogeneous VDM (HVDM), functions that lack a uniform way to treat both continuous and nominal attributes.

The main idea of this paper is to understand the VDM as a way of computing the difference between associations, and to measure such association using correlation coefficients. The new distance function called Fuzzy Correlation-based Difference Metric (FCDM) is able to deal with both continuous and discrete attributes in a conceptually uniform way. More specifically, we use fuzzy sets to model numeric attributes and a uninorm operator to combine their relation with the output classes in a set of instances. Further method, the use of FCDM in conjunction with the $k$-NN Classifier allows us to improve the classification accuracy of this simple instance based learning.

\section{Background}

Ohnishi et al. present in [3] the hypothesis that two types of similarities should be distinguished: a shallow similarity only considering the superficial differences between the objects to be compared and a goal-related deep similarity based on domain knowledge. The VDM is a well-known deep similarity measure which is defined in terms of attribute values that are conditioned by posterior probabilities of a class. Let $\left\{C_{1}, C_{2}, \ldots, C_{c}\right\}$ denote the set of output classes. The following expression shows the local criteria used in the VDM to compare symbolic values $x$ and $y$ for an attribute $a$, where the conditional probability $\mathrm{P}(C \mid x)$ quantifies to what extent we can obtain the class $C$ given the known value $x$.

$$
d_{V D M_{a}}(x, y)=\sqrt{\sum_{l=1}^{c}\left(\mathrm{P}\left(C_{l} \mid x\right)-\mathrm{P}\left(C_{l} \mid y\right)\right)^{2}}
$$

For a given memory of instances the conditional probability can be estimated computing the quantity of instances clasified in the class $C$ has the value $x$ $\left(N_{x, C}\right)$, relative to the appearance $x$ (relative frequency).

The notion of similarity is also strongly related to the theory of fuzzy set. Fuzzy similarity measures and similarity relations have been widely studied but little emphasis has been put in its relation with classification tasks [4. An early variant of a classifier based on fuzzy sets and VDM uses a fuzzy connectionist model to build a local distance function based on relative frequency [5].

\section{Main Results}

Correlation coefficients are mathematical forms of measuring the degree of association or intensity between two random variables. Given a set of $n$ instances, each value for a symbolic attribute would be considered a random variable (dichotomized in this case). Thus, we can use correlation coefficients between binary 
variables (classical statistics 6 ) as a measurement of the association between two symbolic values. Between dichotomic variables the value of the Spearman and Pearson correlation coefficients agree, and it is possible to assure that if there is some dependency this is of linear type. The situations where the conditional probability between two values is zero, the correlation coefficient could give more information. So it is possible to generalize the VDM distance function, which is computed based on the relative frequency, using Pearson's correlation coefficient to quantify the association between two values.

Definition 1. Correlation-Difference metric (CDM). Let $X$ be the attribute domain for a symbolic attribute with $N$ possible values $A_{1}, A_{2}, . ., A_{N}$, which is transformed in $N$ binary variables named $A_{j}, j=1, . ., N$; where $\forall x \in X: A_{k}(x)=$ $1 \Leftrightarrow x=A_{k}$ and $A_{j}(x)=0, \forall j \neq k$. The target attribute domain with $c$ classes $\left\{C_{1}, C_{2}, \ldots, C_{c}\right\}$ is transformed in the same way. The distance between two symbolic values $x, y \in X$ will decrease in the same way that their corresponding binary variables in a given memory of $n$ instances have equal correlation with each possible class $C_{l}, l=1$..c. The distance function $d_{C D M}: X \times X \rightarrow \mathbb{R}^{+}$used only to compare discrete or symbolic values is defined as:

$$
d_{C D M}(x, y)=\sqrt{\sum_{l=1}^{c}\left(r_{x, C_{l}}-r_{y, C_{l}}\right)^{2}}
$$

\subsection{Distance between Fuzzy Sets}

The concept of association between values used before to define the CDM distance function does not make sense for numeric (continuous or discrete) values. We propose to define a fuzzy partition [7 on $X$, that is $\mathcal{P}(X)=\left\{A_{1}, A_{2}, \ldots, A_{N}\right\}$, to be able to use a fuzzy measure of correlation between the defined linguistic labels. Thus, once fuzzy sets are defined for the finite set of instances, each liguistic term can be considered a random variable. In other words, the $j$-th fuzzy set $A_{j} \in \mathcal{P}(X), j=1 \ldots N$ is a random variable taking values $A_{j}\left(x_{i}\right)$ in the interval $[0,1]$ for each example $x_{i}, i=1, \ldots, n$. Now it is possible to use correlation coefficients between two of binary variables, between membership degrees (continuous variables derived from fuzzy variables [8]) or between membership degrees and binary variables (nominal variables previously dichotomized).

In this paper we adopted the concepts from Chiang and Lin [9] to use Pearson's correlation coefficient between fuzzy sets . Let $A \in F(X)$ and $C \in F(Z)$ be two fuzzy sets, a formulation of the Pearson's correlation coefficient between them has been defined as $r_{A, C}: F(X) \times F(Z) \rightarrow[-1,1]$ according to:

$$
r_{A, C}=\frac{\sum_{i=1}^{n}\left(A\left(x_{i}\right)-\bar{A}(x)\right)\left(C\left(z_{i}\right)-\bar{C}(z)\right) /(n-1)}{S_{A} S_{C}}
$$

where $\bar{A}(x)$ is the mean of $A\left(x_{i}\right), i=1 . . n$ while $S_{A}^{2}$ denotes the variance of those values. Now, we are ready to introduce a distance function between fuzzy sets. 
Definition 2. FCDM between fuzzy sets. Let $A$ and $B \in \mathcal{P}(X), C \in \mathcal{P}(Z)$. The distance between fuzzy sets $A$ and $B$ will decrease, in the same way that they have equal correlation with each possible class value. The Fuzzy Correlation-based Difference Metric (FCDM between fuzzy sets), $d_{F C D M}: \mathcal{P}(X) \times \mathcal{P}(X) \rightarrow \mathbb{R}^{+}$is defined by the expression:

$$
d_{F C D M}(A, B)=\sqrt{\sum_{l=1}^{c}\left(r_{A, C_{l}}-r_{B, C_{l}}\right)^{2}}
$$

Expression (4) is a generalization of expression (2) and could be used to compare symbolic and linguistic labels by identifying ordinary sets with their characteristic function. That is, $x \in A \Leftrightarrow A(x)=1 ; x \notin A \Leftrightarrow A(x)=0$. Let $a, b \in X$ and $A, B \in F(X)$. If $A(a)=1$ and $B(b)=1$, then $d_{C D M}(a, b)=$ $d_{F C D M}(A, B)$. In other words, if the elements to be compared have maximum compatibility with one of the defined fuzzy sets (an ordinary set is a particular case), then the results of the distance functions FCDM and CDM are the same. Particularly in this paper we assume a set of hard output classes (i.e. $\left.\left\{C_{1}, C_{2}, \ldots, C_{c}\right\}\right)$.

\subsection{Distance between Elements}

When $x \in X$ is a numerical value and a fuzzy partition has been defined on $X$, a more precise measurement between 0 and 1 of how this value is represented by each linguistic term $A_{j} \in F(X)$ is given by the corresponding membership degree $A_{j}(x)$. With the purpose of differentiating the comparison between elements starting from expression (44), the special kind of aggregation used in the MYCIN expert system to handle the certainty [10] is closely related. More specifically, the uninorm $U:[-1,1]^{2} \rightarrow[-1,1]$ in expression (5) is used, because of the certainty factor for a hypothesis can take value within the interval $[-1,1]$ with the desired meaning.

$$
U(x, y)= \begin{cases}x+y-x y & \text { if } x, y \geq 0 \\ x+y+x y & \text { if } x, y<0,|x|,|y| \leq 1 \\ (x+y) /(1-\min (|x|,|y|)) & \text { otherwise }\end{cases}
$$

Note that the previous definition is not clear in the points $(-1,1)$ and $(1,-1)$, though it is understood that the result is -1 . Since the uninorm $U$ is associative, it can be extended to several arguments in an unambiguous way, i.e. to a number of predictive attributes, as follows: $\amalg_{j=1}^{n} y_{j}=U\left(y_{1}, U\left(y_{2}, \ldots, U\left(y_{n-1}, y_{n}\right)\right)\right)$.

Definition 3. (FCDM between elements). Let $\mathcal{P}(X)=\left\{A_{1}, A_{2}, \ldots, A_{N}\right\}$ be a fuzzy partition defined on $X$. The strength of the relationship between an element $x \in X$ and the class $C$ is computed as the weighted aggregation of the correlations between each fuzzy set $A_{j} \in F(X)$ and $C$, weighted by the membership degrees $A_{j}(x)$ of the given value $x$ as:

$$
S(x, C)=\amalg_{j=1}^{N}\left(A_{j}(x) \cdot r_{A_{j}, C}\right)
$$


to define the Fuzzy Correlation-based Difference Metric (between elements) $d_{F C D M}: X \times X \rightarrow \mathbb{R}^{+}$we use the expression:

$$
d_{F C D M}(x, y)=\sqrt{\sum_{l=1}^{c}\left(S\left(x, C_{l}\right)-S\left(y, C_{l}\right)\right)^{2}}
$$

We recall that if discrete or symbolic values (crisp sets) are compared, expression (77) turns into expression (44). Besides, the comparison in presence of missing value is implicit in the criterion defined in the expression above. In other words, taking into account the relations of both fuzzy and possibility theory [11, the membership degree $A_{j}(x)$ is the compatibility degree of value $x$ with linguistic term $A_{j}$. Thus, when the value of an attribute a is unknown $(x=$ ? or $y=$ ), the maximum compatibility with each fuzzy set defined for its attribute is assumed. That is, the membership degree $A_{j}(?)=1, j=1, \ldots N$ in expression (6).

\section{Experimental Results and Discussion}

The $k$-NN classifier bases its answers on similarity between the query and the training instances (or examples $e$ ). The generalization is postponed until a request is received (lazy learning). Given a new query $q=\left(q_{1}, q_{2}, \ldots, q_{m-1}\right)$, the $k$ most similar cases used to predict the output class $\left(q_{m}\right)$ can be retrieved by using the FCDM as the local distance function instead of the HEOM in the standard $k$-NN. The nearest neighbor rule decides that the query $q$ belongs to the category of the majority class of the nearest neighbors. The test bed employed for comparison was the Waikato Environment for Knowledge Analysis (WEKA) 12 . The new classifier was easily implemented as a part of the WEKA package. It was necessary to add a new type of data (called LINGUISTIC), the new filter to obtain a fuzzy partition for numeric attributes, and the new distance function FCDM as a local criterion of comparison.

Trapezoidal membership functions to represent numeric attributes using fuzzy sets were selected. Each numeric attribute is discretized using the supervised discretization method of Fayyad and Irani [13, allowing us to define the corresponding membership function. The parameters of the membership functions are set in such a way guaranteeing that two consecutive functions have as cut point the limit of the interval and each individual fuzzy set has the support equal to half of the interval.

In the experiments presented, 19 data sets from UCIMLR [14 having both numeric and symbolic predictive attributes, one target attribute and 11 containing missing values (names in bold). The percentage of cases correctly classified (accuracy) was considered as measure of the performance. Each experiment was repeated ten times for each dataset using 10-fold cross validation.

The second column in Table 1 presents the performance mean for each data set using the distance proposed here considering $k=1$. The results with the other 
Table 1. Accuracy measures obtained with the 1-NN and several distance functions

\begin{tabular}{|l|c|c|c|c|c|}
\hline Data set name & FCDM & HEOM & HVDM & IVDM & DVDM \\
\hline \hline 1. Diabetes & 69.05 & 70.31 & 71.09 & 69.28 & $\mathbf{7 1 . 8 9}$ \\
\hline 2. Glass & 70.53 & 70.52 & $\mathbf{7 2 . 3 6}$ & 70.54 & 56.06 \\
\hline 3. Ionosphere & 90.43 & 86.33 & 86.32 & 91.17 & $\mathbf{9 2 . 6}$ \\
\hline 4. Iris & 94.87 & $\mathbf{9 5 . 3 3}$ & 94.67 & 94.67 & 92 \\
\hline 5. Liver Disorders & 58.84 & $\mathbf{6 3 . 4 7}$ & 62.92 & 58.23 & 55.04 \\
\hline 6. Sonar & 81.44 & 86.6 & $\mathbf{8 7 . 0 2}$ & 84.17 & 78.45 \\
\hline 7. Vehicle & $\mathbf{7 1 . 1 8}$ & 70.22 & 70.93 & 69.27 & 63.72 \\
\hline 8. Wine & 97.17 & 95.46 & 95.46 & $\mathbf{9 7 . 3 8}$ & 94.38 \\
\hline 9. Wbc & $\mathbf{9 5 . 6 2}$ & 95.28 & 94.99 & 95.57 & 95.57 \\
\hline 10. Monks-1 test & $\mathbf{8 1 . 9 7}$ & 69.43 & 68.09 & 68.09 & 68.09 \\
\hline 11. Monks-2 test & 94.41 & 54.65 & $\mathbf{9 7 . 5}$ & $\mathbf{9 7 . 5}$ & $\mathbf{9 7 . 5}$ \\
\hline 12. Monks-3 test & $\mathbf{1 0 0 . 0 0}$ & 78.49 & $\mathbf{1 0 0 . 0 0}$ & $\mathbf{1 0 0 . 0 0}$ & $\mathbf{1 0 0 . 0 0}$ \\
\hline 13. Thyroid (allbp) & $\mathbf{9 5 . 9 8}$ & 94.89 & 95 & 95.32 & 94.86 \\
\hline 14. Thyroid (allhyper) & 97.34 & 97.00 & 96.86 & $\mathbf{9 7 . 8 6}$ & 96.93 \\
\hline 15. Thyroid (allhypo) & $\mathbf{9 8 . 2 6}$ & 90.39 & 90.29 & 96.07 & 89.36 \\
\hline 16. Thyroid (allrep) & 96.86 & 96.14 & 96.11 & $\mathbf{9 8 . 4 3}$ & 96.86 \\
\hline 17. Credit-app & 80.12 & $\mathbf{8 1 . 0 1}$ & 80.87 & 80.14 & 80.14 \\
\hline 18. Anneal & $\mathbf{9 9 . 4 2}$ & 94.61 & 94.61 & 96.11 & 94.99 \\
\hline 19. Hepatitis & 80.50 & 77.5 & 76.67 & $\mathbf{8 2 . 5 8}$ & 80.58 \\
\hline Accuracy \% All datasets & $\mathbf{8 7 . 0 5}$ & 82.51 & 85.88 & 86.44 & 84.16 \\
\hline Wins \% & $\mathbf{4 2 \%}$ & $5 \%$ & $21 \%$ & $21 \%$ & $21 \%$ \\
\hline Accuracy Datasets 1-8\% & 79.19 & 79.78 & 80.10 & 79.34 & 75.52 \\
\hline Wins \% & $25 \%$ & $13 \%$ & $25 \%$ & $13 \%$ & $25 \%$ \\
\hline Significance levels & - & 0.019 & 0.185 & 0.706 & 0.009 \\
\hline Accuracy \% Datasets 9-19 & $\mathbf{9 2 . 7 7}$ & 84.49 & 90.09 & 91.61 & 90.44 \\
\hline Wins \% & $\mathbf{6 0 \%}$ & $0 \%$ & $20 \%$ & $30 \%$ & $20 \%$ \\
\hline Significance levels & - & 0.001 & 0.019 & 0.379 & 0.070 \\
\hline
\end{tabular}

distance functions were taken from [2]. The "Wins" rows indicate the proportions of data sets in which each distance function obtained the highest accuracy according to all distance functions considered in the experiment.

Note that by using FCDM the classifier achieved the general behavior with all data sets higher for both accuracy and percentage of wins measures. When only dates sets with missing values or symbolic attributes are considered (rows 9-19) the results are much better in favor of the distance function proposed (see last rows in the table above). Additionally, three hypotheses were investigated for statistical analysis:

$T_{1}$ : The new distance function improves the results achieved using standard $k$-NN.

$T_{2}$ : The uniform way to extend VDM by using fuzzy sets presented here does not shows a significant difference in performance compared to other extensions based on crisp values. 


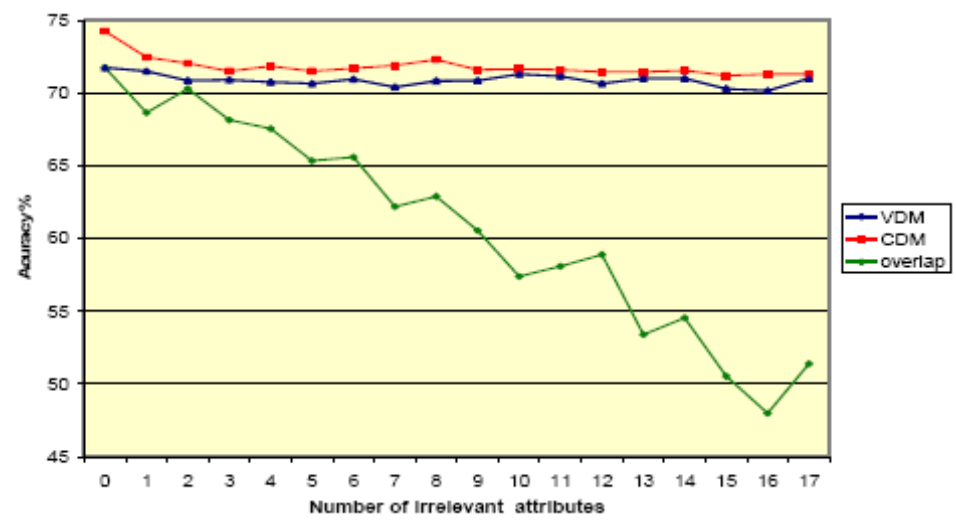

Fig. 1. LED artificial results increasing the number of irrelevant attributes

$T_{3}$ : The FCDM shows a significant improvement over the VDM when missing values or symbolic data is used.

The non parametric the Wilcoxon signed-rank test was used to compare the model proposed with the rest of them. The significance is based in 10000 sampled tables with Monte Carlo simulation techniques and a $99 \%$ confidence interval (see rows with significance levels in Table 10.

The results indicate a significant improvement for the distance function proposed in this paper (FCDM) compared to the function used by standard $k$-NN (HEOM) (significance of the test 0.001 when data sets contain missing values and 0.019 in other case). With respect to the second trend, no significant differences are found in comparison to the HVDM and IVDM on datasets without missing values (significance of the test 0.185 and 0.706 respectively). That is to say, there are not enough elements to conclude the same as for the HEOM. Nevertheless, the same experiment on data sets with missing values or symbolic data corroborates a significant improvement of the FCDM over the VDM (significance of the test 0.019).

We also wanted to evaluate the behavior of FCDM in presence of irrelevant attributes, since it is considered a generalization of the VDM [15. For this reason, the metrics were evaluated on the well-known 24-attribute LED display problem. Figure 1 shows the classification accuracies achieved increasing the number of irrelevant attributes from zero to seventeen, after repeating each test ten times. Since the LED data set only has symbolic attributes, the HVDM and IVDM are identical to the VDM, the HEOM is really referred to Overlap distance function. As the number of irrelevant attributes increased, the classification accuracies of the overlap metric fell from $71.75 \%$ to $51.40 \%$ while both CDM and VDM showed no signs of degradation as the number of irrelevant attributes increased.

\section{Conclusions and Future Work}

The Fuzzy Correlation-based Difference Metric proposed in this paper (FCDM) is a uniform way to extend the VDM using fuzzy sets to model numeric attributes 
and based on correlation coefficients. It allows improving the performance of the Nearest Neighbor classifier compared to other distance functions proposed before, mainly when missing values or symbolic data are used. Besides, the FCDM preserves the advantages of the VDM showing no degradation of performance in presence of irrelevant attribute. Next, we need to study the influence of different ways of creating fuzzy partitions on the classifier performance. Another interesting question for future work is to test FCDM with other similarity-based methods.

\section{References}

1. Stanfill, C., Waltz, D.: Towarder memory-based reasoning. Communication of the ACM 29, 1213-1228 (1986)

2. Wilson, R., Martinez, T.: Improved heterogeneous distance functions. Journal of Artificial Intelligence Research 6, 1-34 (1997)

3. Ohnishi, H., Suzuki, H., Shigemasu, K.: Similarity by feature creation: Reexamination of the asymmetry of similarity. In: 16th Annual Conference of Cognitive Science Society. Lawrence Erlbaum, Mahwah (1994)

4. Zwick, R., Carlstein, E., Budescu, D.: Measures of similarity among fuzzy sets: a comparative analysis. International Journal of Approximate Reasoning 1(2), 221242 (1987)

5. Rodriguez, Y., Garcia, M., De Baets, B., Morell, C., Bello, R.: A connectionist fuzzy case-based reasoning model. In: Gelbukh, A., Reyes-Garcia, C.A. (eds.) MICAI 2006. LNCS (LNAI), vol. 4293, pp. 176-185. Springer, Heidelberg (2006)

6. Siegel, S., Castellan, N.: Nonparametric Statistics for the Behavioral Sciences. McGraw-Hill, New York (1988)

7. Ruspini, E.: A new approach to clustering. Information and Control 15, 22-38 (1969)

8. Zadeh, L.: The concept of a linguistic variable and its applications to approximate reasoning, parts I, II. Information Sciences 8, 199-251, 301-357 (1975)

9. Chiang, D., Lin, N.: Correlation of fuzzy sets. Fuzzy Sets and Systems 102(2), 221-226 (1999)

10. Tsadiras, A., Margaritis, K.: The mycin certainty factor handling function as uninorm operator and its use as a threshold function in artificial neurons. Fuzzy Sets and Systems 93(3), 263-274 (1998)

11. Dubois, D., Nguyen, H., Prade, H.: Possibility theory, probability and fuzzy sets: misunderstandings, bridges and gaps. In: Fundamentals of Fuzzy Sets, pp. 343-438. Kluwer Academic Publishers, Boston (2000)

12. Witten, I., Frank, E.: Data Mining: Practical Machine Learning Tools and Techniques, 2nd edn. Morgan Kaufmann Series in Data Management Systems. Morgan Kaufmann, San Francisco (2005)

13. Fayyad, U., Irani, K.: Multi-interval discretization of continuous-valued attributes for classification learning. In: IJCAI 1993 (1993)

14. Murphy, P., Aha, D.: Uci repository of machine-learning databases, http://www.ics.uci.edu/ mlearn/mlrepository.htm

15. Payne, T., Edwards, P.: Implicit feature selection with the value difference metric. In: ECAI 1998: 13th European Conference on Artificial Intelligence (1998) 